\title{
Group schema therapy for eating disorders: study protocol
}

\author{
Fiona Calvert ${ }^{1,2}$, Evelyn Smith ${ }^{2^{*}}$ (D), Rob Brockman ${ }^{3}$ and Susan Simpson ${ }^{4}$
}

\begin{abstract}
Background: The treatment of eating disorders is a difficult endeavor, with only a relatively small proportion of clients responding to and completing standard cognitive behavioural therapy (CBT). Given the prevalence of co-morbidity and complex personality traits in this population, Schema Therapy has been identified as a potentially viable treatment option. A case series of Group Schema Therapy for Eating Disorders (ST-E-g) yielded positive findings and the study protocol outlined in this article aims to extend upon these preliminary findings to evaluate group Schema Therapy for eating disorders in a larger sample $(n=40)$.

Methods/design: Participants undergo a two-hour assessment where they complete a number of standard questionnaires and their diagnostic status is ascertained using the Eating Disorder Examination. Participants then commence treatment, which consists of 25 weekly group sessions lasting for $1.5 \mathrm{~h}$ and four individual sessions. Each group consists of five to eight participants and is facilitated by two therapists, at least one of who is a registered psychologist trained on schema therapy. The primary outcome in this study is eating disorder symptom severity. Secondary outcomes include: cognitive schemas, self-objectification, general quality of life, self-compassion, schema mode presentations, and Personality Disorder features. Participants complete psychological measures and questionnaires at pre, post, six-month and 1-year follow-up.

Discussion: This study will expand upon preliminary research into the efficacy of group Schema Therapy for individuals with eating disorders. If group Schema Therapy is shown to reduce eating disorder symptoms, it will hold considerable promise as an intervention option for a group of disorders that is typically difficult to treat.
\end{abstract}

Trial registration: ACTRN12615001323516. Registered: 2/12/2015 (retrospectively registered, still recruiting).

\section{Background}

The treatment of eating disorders is a difficult endeavor, with only a relatively small proportion of clients responding to standard cognitive behavioural therapy (CBT). Less than half of those with bulimia nervosa $(\mathrm{BN})$ have recovered at follow-up after receiving CBT $[17,18,23]$ and research supporting cognitive-behavioural treatment for anorexia nervosa (AN) is limited, with no clear indication of improvement in this population $[6,8]$. Approximately $50 \%$ of patients with eating disorders continue to be highly symptomatic at 60 -week follow-up following transdiagnostic CBT [16]. Further, treatment dropout rates are high amongst individuals with eating disorders $[9,43]$ with one literature review reporting an

\footnotetext{
* Correspondence: evelyn.smith@westernsydney.edu.au

${ }^{2}$ School of Social Sciences and Psychology, Western Sydney University, 1795 Locked bag, Penrith, NSW, Australia

Full list of author information is available at the end of the article
}

average drop-out rate of between 20 and $51 \%$ in inpatient settings and between 29 and $73 \%$ in outpatient settings [20].

The treatment of eating disorders is especially complicated by a high level of co-morbidity [3]. Approximately $69 \%$ of individuals with eating disorders may meet DSM IV (APA, 1994) diagnostic criteria for a personality disorder and $93 \%$ of these clients may also have other comorbidity including anxiety and substance use disorders. Eating disorders are also associated with the presence of rigid personality features, which increases clinical complexity and is associated with poorer treatment outcomes [22, 26, 46]. Eating disorders have also been linked to a range of trauma-related risk factors, including childhood abuse and neglect, which may also be mediated by personality disorder diagnoses [5]. Individuals with eating disorders also commonly experience complex and difficult-to-treat symptomatology including 
dissociation, perfectionism, compulsive pathology, rigid thinking patterns $[28,30,38,49]$ and high levels of shame [7].

Given the prevalence of co-morbidity and complex personality traits in this population, it is important to consider the deeper belief systems underlying eating disorder presentations. Schema Therapy ([53]/1999) is becoming an increasingly popular psychological model for working with individuals with complex mental health and personality difficulties. Schema Therapy combines aspects of cognitive, behavioral, experiential, interpersonal and psychoanalytic therapies into one integrative and unified model [1]. The schemas that are targeted in treatment are enduring and self-defeating patterns that typically begin early in life. These patterns consist of negative/dysfunctional thoughts and feelings which have been repeated and elaborated upon, and pose obstacles for accomplishing one's goals and getting one's needs met [40]. These schemas are perpetuated behaviorally through the coping styles of schema maintenance, schema avoidance, and schema compensation. The Schema therapy model of treatment is designed to help the person break these negative patterns of thinking, feeling and behaving and develop healthier alternatives to replace them [1].

The evidence for schema therapy for individuals with complex mental health difficulties is growing. This approach has been applied, in both individual and group forms, to a wide variety of clinical disorders, including, borderline personality disorder $[19,21]$ and chronic depression [11, 34, 41, 42]. A recent stringent systematic review found medium to large effect sizes for schema therapy in the treatment of a range of psychological conditions [35]. Attention has recently been given to the applicability of Schema Therapy to individuals with eating disorders (Pugh, 2015). Evidence suggests that maladaptive schemas are more strongly held by individuals with anorexia and bulimia nervosa compared to normal controls [30]. Preliminary data [33, 38] supports the notion that it is the schema processes that are engaged in an attempt to avoid intolerable emotional states associated with these schemas that in fact determine whether an individual will manifest restrictive or bulimic eating pathology. Whereas restrictive eating pathology may be a compulsive behavior developed to prevent schemas being triggered at all (schema compensation), bulimic pathology may function alongside other impulsive behaviors as a method of escaping schema-related affect once schemas have already been triggered (schema avoidance) [33, 38].

Schema therapy has been used in individuals with eating disorders in one preliminary study [44]. Simpson et al. examined the use of Group Schema Therapy for Eating Disorders (STE-g) in a case series of eight participants with chronic eating disorders and high levels of comorbidity. Treatment was comprised of 20 sessions which included cognitive, experiential, and interpersonal strategies, with an emphasis on behavioral change. Clinically significant change was observed from pre-treatment to six-month follow-up for eating disorder severity $(d=1.70)$, global schema severity $(d=1.59)$, shame $(d=0.91)$, and anxiety $(d=1.53)$. Clinically significant change in eating disorder severity at follow-up was also shown for the majority of completers (six participants out of eight completed the full treatment program). Self-report feedback suggested that group factors may catalyze the change process in schema therapy by increasing perceptions of support and encouragement to take risks and try out new behaviors, whilst providing a de-stigmatizing and deshaming therapeutic experience [44].

The present study aims to extend upon the preliminary findings of Simpson et al. [44] to evaluate Schema Therapy in a large eating disordered sample $(n=40)$, in terms of reduction of symptoms, and assess feasibility, acceptability and predictors of outcomes. We aim to conduct 6 groups of schema therapy in two locations: 1) University of South Australia in Adelaide Australia and 2) Western Sydney University in Sydney Australia. The study will examine whether Schema Therapy reduces eating disorder symptoms and improves psychological wellbeing and quality of life, both at post treatment and follow-up.

\section{Method \\ Participants}

Approximately 40 participants will be recruited into the study. All participants will be females aged 18 years and over, meeting Diagnostic and Statistical Manual of Mental Disorders, 5th Edition criteria for an eating disorder, following a transdiagnostic approach (Fairburn, et al., 2003). Participants are recruited via word of mouth, letters to clinicians and advertisements through Facebook as well as support organisations such as The Butterfly Foundation. Participants are provided with information about the study and, if they agree to participate, are required to give written consent. Full disclosure of the purpose of the study, the potential benefits and risks associated with participation, and the confidential nature of information obtained in the study is explained to participants.

\section{Inclusion/ exclusion criteria}

Participants with active psychotic symptoms, high suicide risk or current crisis status (self-disclosed at baseline), a BMI of less than 14, intellectual disability, and those who are consuming large amounts of alcohol/ drugs are excluded from the study. All participants must have a general practitioner involved in their care to monitor their physical health in order to participate in this research. 


\section{Overall study design}

This study will be an uncontrolled single group repeated measures design. Ethics approval was secured and participants provided informed consent to participate. Participants are first screened over the phone to ensure suitability for the group. If they agree to participate in the group, they then undergo a two-hour assessment where they complete a number of standard questionnaires and their diagnostic status is ascertained. Participants then commence treatment which consists of 25 weekly group sessions lasting for $1.5 \mathrm{~h}$. Participants are also provided with four individual sessions that they can book with one of the therapists whenever they want. Each group consists of six to eight participants and is facilitated by two therapists, at least one of whom is a registered psychologist with training on schema therapy, and supervised by a schema therapist. Participants complete psychological measures and questionnaires at pre, post, 6 month and 1 year follow-up.

\section{Measures}

This study utilises a battery of assessments conducted at baseline, mid-treatment, end-of-treatment, and six- and twelve-month follow-up points. These assessments are conducted by fully registered psychologists who have received specialized training in the administration of standardized eating disorder measures. Table 1. Provides a summary of these assessments.

\section{Eating Disorder Examination (EDE; [15])}

The EDE is a structured, investigator-based interview that measures the severity of symptoms of eating disorders. The scale can be used to ascertain an individual's eating disorder diagnosis, as is the purpose of the

Table 1 Assessments conducted at different time points

\begin{tabular}{|c|c|c|c|c|c|c|}
\hline Measure & Baseline & Weekly & Mid & Post & 6 months & 12 months \\
\hline EDE & $x$ & & & $x$ & & \\
\hline EDE-Q & $x$ & x (abbrev) & $x$ & $x$ & $x$ & $x$ \\
\hline YSQ-SF & $x$ & & $x$ & $x$ & $x$ & $x$ \\
\hline SMI & $x$ & & $x$ & $x$ & $x$ & $x$ \\
\hline WHO-5 & $x$ & & $x$ & $x$ & $x$ & $x$ \\
\hline CORE-10 & $x$ & & $x$ & $x$ & $x$ & $x$ \\
\hline SCS-SF & $x$ & & $x$ & $x$ & $x$ & $x$ \\
\hline SATAQ & $x$ & & $x$ & $x$ & $x$ & $x$ \\
\hline MCMI-III & $x$ & & & $x$ & & \\
\hline BSL-23 & $x$ & & & $x$ & $x$ & $x$ \\
\hline
\end{tabular}

Notes: $E D E=$ Eating Disorder Examination; $E D E-Q=$ Eating Disorder ExaminationQuestionnaire; YSQ-SF = Young Schema Questionnaire, short form; SMI = Schema Mode Inventory; WHO-5 = World Health Organisation-Five Well-Being Index: CORE-10 = Clinical Outcomes in Routine Evaluation-Outcome Measure; SCS = Self-Compassion Scale-Short Form; SATAQ = Sociocultural Attitudes Towards Appearance Questionnaire; MCMI-III = Millon Clinical Multiaxial Inventory- III; $B S L-23=$ Borderline Symptoms List measure in the current study. The EDE has been shown to have excellent reliability when administered by trained examiners [12].

\section{Eating Disorder Examination- Self-Report Questionnaire Version (EDE-Q; [14])}

The EDE-Q is a 36-item self-report questionnaire for the assessment and diagnosis of eating disorders. The EDE-Q yields four subscale scores-Restraint, Eating Concern, Weight Concern, and Shape Concern-as well as a global score, which is an average of all four subscales. The EDE-Q has been shown to have good convergent validity $[10,14]$. Acceptable internal consistency and test-retest reliability have also been demonstrated [32, 37].

\section{Young Schema Inventory- Short Form (YSQ-SF; [52])}

The YSQ-SF is a self-report measure used to assess 15 different maladaptive schemas (emotional deprivation, abandonment, mistrust/abuse, social alienation, defectiveness, incompetence, dependency, vulnerability to harm, enmeshment, subjugation of needs, self-sacrifice, emotional inhibition, unrelenting standards, entitlement, and insufficient self-control). The scale consists of 75 items rated from one (completely untrue of me) to six (describes me perfectly). The scale has been shown to have good psychometric properties $[25,50]$.

Schema Mode Inventory-Short Form (SMI; [31])

The SMI measures the presence of 14 schemas modes: Vulnerable Child, Angry Child, Enraged Child, Impulsive Child, Undisciplined Child, Happy Child, Compliant Surrender, Detached Protector, Detached Self-Soother, Self-Aggrandizer, Bully and Attack, Punitive Parent, Demanding Parent and Healthy Adult modes. The questionnaire consists of 118 items which are given frequency ratings using a Likert scale ranging from one (never or hardly ever) to six (always). An overall score is calculated from the scale sum score divided by the number of items in that scale. The short form of the SMI has been shown to have acceptable internal consistencies amongst the 14 subscales (Cronbach $\alpha$ 's from .79 to .96 ) as well as adequate test-retest reliability and moderate construct validity [31].

World Health Organisation-Five Well-Being Index (WHO-5; [51]) The WHO-5 is an assessment of general wellbeing consisting of five statements (e.g. I have felt cheerful and in good spirits and I have felt calm and relaxed), which participants rate on a six-point scale (from never to always), with a possible total score varying from 0 to 25 . Higher scores on the WHO-5 reflect better well-being. 
Clinical Outcomes in Routine Evaluation-Outcome Measure (CORE-10; [2])

The CORE-10 is a self-report measure of general psychological distress. The CORE-10 includes 10-items which the respondent rates on a five-point Likert scale (from not at all to most or all of the time), for example $I$ have felt tense, anxious or nervous and I have felt panic or terror. The CORE-10 has been shown to have good internal reliability $(\alpha=.90)$ and a correlation of.94 with the CORE-OM [2].

\section{Self-Compassion Scale- Short Form (SCS-SF; [39])}

The SCS-SF is a 12-item, self-report scale which assesses the positive and negative aspects of the three main components of self-compassion: Self-Kindness (e.g., When I'm going through a very hard time, I give myself the caring and tenderness I need) versus Self-Judgment (e.g., I'm disapproving and judgmental about my own flaws and inadequacies); Common Humanity (e.g., When I feel inadequate in some way, I try to remind myself that feelings of inadequacy are shared by most people) versus Isolation (e.g., When I fail at something that's important to me, I tend to feel alone in my failure); and Mindfulness (When something upsets me I try to keep my emotions in balance) versus Over-Identification (When I'm feeling down I tend to obsess and fixate on everything that's wrong). Responses are given on a five-point scale ranging from one (almost never) to five (almost always). A total self-compassion score is calculated as a mean of all items and higher scores correspond to higher levels of self-compassion. The SCS-SF has good psychometric properties, with high internal consistency $(\alpha=.85$; [45]) and a very high correlation with the long form of the SCS [39].

\section{Sociocultural Attitudes Towards Appearance Questionnaire-} Internalization subscale (SATAQ; [24])

The SATAQ is a 14-item inventory assesses women's recognition and acceptance of societally prescribed standards of physical appearance, particularly the thin ideal. The 8-item Internalization subscale is used in the present study and measures the extent to which the individual personally accepts these standards. Statements such as I tend to compare my body to people in magazines and on $T V$ are rated from one (completely disagree) to five (completely agree). The SATAQ converges satisfactorily with other measures of body image and eating disturbance [24, 47].

\section{Millon Clinical Multiaxial Inventory- III [36]}

The MCMI-III is a psychological assessment that provides information on longstanding personality patterns and clinical symptomatology. The tool consists of 175 items that are scored on a True/False basis that are scored to produce 28 clinical subscales. Reliability and validity studies on the MCMI indicate that is generally a psychometrically sound instrument. The scale demonstrates good internal consistency with alpha coefficients of above.80 for the majority of the scales (manual). Test-retest reliability has been shown to be moderate to high $[13,29]$.

\section{Borderline Symptoms List (BSL-23; [4])}

The BSL-23 is a questionnaire used to assess the degree of symptoms of BPD, such as poor self-esteem, dysphoric emotions, suicidal intention and impulsive behaviors. The scale consists of 23 items (for example: $I$ experienced stressful inner tension and I wanted to punish myself) rated on a five-point Likert scale from 0 (not at all) to 4 (very strong). A total score is obtained by summing responses and higher scores represent more severe BPD symptomatology. The BSL-23 has good psychometric properties with high internal consistency $(\alpha=0.94-0.97)$ and the ability to discriminate personality disorder patients from patients with other clinical symptomatology (mean effect size of 1.13; [4]).

\section{Primary outcomes}

Eating disorder symptom severity is the primary outcome of this, as measured by the EDE and EDE-Q. The EDE will be used for pre to post, but due to limited resources only the EDE-Q will be completed at follow-up.

\section{Secondary outcomes}

The secondary outcomes measured in this study include: cognitive schemas (measured using the YSQ); self-objectification (measured using the SATAQInternalisation); general quality of life (WHO-5 and CORE-10); self-compassion (SCS-SF); schema mode presentations (measured using the SMI); and Personality Disorder features (measured using the MCMI-III and the BSL-23).

\section{Intervention}

The schema therapy eating disorder group (STE-g; [44]) was based on the schema mode model, with some components drawing on the schema-therapy treatment program: "Schema Focused Therapy in a Group Setting" [48]. The program consists of twenty-five 90-min sessions. All participants are provided with a patientversion workbook which corresponds with the treatment manual. The first part of the group focuses on schema psychoeducation and schema-focused cognitive behavioral strategies which help participants to identify and start challenging their schemas, whilst working on behavioral change both within and outside the group. This model assists participants to develop an individualised formulation of their own difficulties using a schema 
therapy framework. The highest schemas and modes are identified for each patient on the basis of their scores on relevant measures. Each session is recorded and group members are strongly encouraged to come in to watch the video should they miss a session. This is to ensure that participants do not miss any educational material, and retain a sense of connectedness with the group.

Particular emphasis is placed on linking eating behaviors to the schema modes, so that participants are able to learn about the origins and coping functions associated with their own pattern of symptoms. Avoidant coping in the group is addressed frequently by labeling the "Detached Protector" mode when it is identified either by group leaders or by other participants (i.e., both in the context of the group and when talking about eating behavior which took place between sessions). Use of over-compensatory behaviors to avoid emotions is also identified and group members are encouraged to point this out to each other empathically as the group progresses. Due to the lack of emotional awareness and emotional tolerance within the group, participants are encouraged to learn to express empathy and to ask for their emotional needs to be met within the context of the group, rather than detaching from emotional needs through eating behaviors. Schema-focused mindfulness meditation [27], is incorporated to increase awareness of urges to carry out eating disordered behaviors, modes and associated triggers and to facilitate emotional regulation.

Participants are helped to identify the origins of their negative body-image, and to link these to the development of particular modes. Body-image restructuring work involves participants learning to recognise the way in which their perception and visceral sense of their bodies differs from the perspective of their most prominent modes, and developing a compassionate "Healthy Adult" mode which is accepting and nurturing toward the body. Mode dialogues are used to enable group members to repeatedly coach each other to send the "Critical" mode away when it expresses negative assertions about the body or self (e.g. "You are ugly, fat, and nobody really likes you"). This gives group participants the opportunity to practice being in Healthy Adult mode firstly for others and then for themselves. Flashcards are used to reinforce what is learned in sessions and to help participants to challenge modes and resist eating disordered behavior between sessions. In the four individual sessions the therapist ensure that the participant understand the schema model, manages crisis, and engages in schema therapy.

\section{Data analysis}

Within-patient standardized effect size information (i.e., Cohen's d) from baseline to end-of-treatment, and from baseline to follow-up will be calculated for eating psychopathology, and all secondary outcomes. Clinical outcome data will be analysed using intention to treat criteria. Data from outcome measures will be analysed with paired sample T-test. To assess group differences between the two treatment sites, independent $t$-tests will be used. The effect size of outcome measures will be compared to the effect size reported for previous studies CBT for eating disorders. The criterion used for statistical significance will be $p<0.05$. Sample size was estimated assuming $80 \%$ power, significance level at 0.05 , and an effect size of $0.5 ; 26$ people are needed. Assuming a $50 \%$ drop out as documented in previous CBT trials, we will need around 40 people.

At the completion of the treatment, a random set of participants will be invited to provide qualitative feedback on their experiences of the group treatment. These interviews will be one-to-one, and conducted either face-to-face, or via telephone, and are expected to last for between 30 and $60 \mathrm{~min}$. Transcripts of these interviews will be made and the data will be analysed using a thematic analyses approach. This information will be used to provide preliminary information about treatment efficacy and acceptability, and inform the design of future studies.

\section{Discussion}

This study will expand upon preliminary research of group Schema Therapy for individuals with eating disorders [44]. If group Schema Therapy is shown to reduce symptoms for this population, it will hold considerable promise as an intervention option for a group of disorders that is typically difficult to treat.

As this is an uncontrolled study it is limited by not having a proper comparison to control for confounding variables. We initially designed this study with a control group, group Cognitive Behaviour Therapy (CBT), but then were unable to recruit participants for the CBT group in Adelaide, and participants only wanted to take part of the research if they were in the schema therapy group. This was mostly due to the fact that Adelaide is a small city and referrals were usually received for severe cases, as most of the participants so far had completed CBT before. We do wish to conduct a randomized controlled trial comparing schema therapy to CBT, but that will need to be conducted in Sydney.

Since the groups are transdiagnostic this has a few advantages and disadvantages. First, the transdiagnostic approach for eating disorders is well established, it helps having different eating disorders as this will provide variety in the group on schemas and modes, which might facilitate engagement. However, within the group people might feel very dissimilar to each other within a transdiagnostic approach, and the psychologists running the 
groups will keep this in mind to bring unity to the group by articulating commonalities among participants.

This study will be the first to modify schema therapy to fit the eating disorders sample and to deliver it to this group. This uncontrolled study will establish proof of concept and assess feasibility, acceptability and potential harms. To date, the study has completed four 25-week group treatments and has commenced a further two groups.

\section{Acknowledgements}

We would like to thank Western Sydney University for the funding for this study, titled Western Sydney University Women's Fellowship. We would also like to thank the participants and the psychologists involved in this study (Sharon Stern, Claudia Mendez, Charlotte Whittingham, Kate Veremeenko, Penelope Richards, Sally-ann Skewes, Rachel Samson, Nicole Middleton).

\section{Authors' contributions}

ES and SS designed the treatment protocol and secured ethics; RB reviewed the protocol; FC wrote the first draft; ES, SS and RB edited the manuscript. All authors read and approved the final manuscript.

\section{Competing interests}

The authors declare they have no competing interests.

\section{Publisher's Note}

Springer Nature remains neutral with regard to jurisdictional claims in published maps and institutional affiliations.

\section{Author details \\ ${ }^{1}$ School of Psychology, University of Wollongong, Wollongong, NSW, Australia. ${ }^{2}$ School of Social Sciences and Psychology, Western Sydney University, 1795 Locked bag, Penrith, NSW, Australia. ${ }^{3}$ University of Technology Sydney, Ultimo, NSW, Australia. ${ }^{4}$ School of Psychology, Social Work \& Social Policy, University of South Australia, Adelaide, SA, Australia.}

Received: 21 August 2017 Accepted: 13 December 2017

Published online: 09 January 2018

\section{References}

1. Bamelis LLM, Evers SMAA, Spinhoven P, Arntz AR. Results of a multicenter randomized controlled trial of the clinical effectiveness of schema therapy for personality disorders. Am J Psychiatr. 2014;171:305-22.

2. Barkham M, Bewick B, Mullin T, Gillbody S, Connell J, Cahill J, Evans C. The CORE10: a short measure of psychological distress for routine use in the psychological therapies. Counselling and Psychotherapy Research: Linking research with Practice. 2013;13:3-13. https://doi.org/10.1080/14733145.2012.729069.

3. Blinder BJ, Cumella EJ, Sanathara VA. Psychiatric comorbidities of female inpatients with eating disorders. Psychosom Med. 2006:68:454-62.

4. Bohus M, Kleindienst N, Limberger M, Stieglitz R, Domsalla M, Chapman A, Wolf $\mathrm{M}$. The short version of the borderline symptom list (BSL-23): development and initial data on psychometric properties. Psychopathology. 2009:42:32-9.

5. Brewerton TD. Eating disorders, trauma and comorbidity: focus on PTSD. Eat Disord. 2007:15:285-304

6. Bulik C, Berkman N, Brownley K, Sedway J, Lohr K. Anorexia nervosa treatment: a systematic review of randomized controlled trials. Int J Eat Disord. 2007:40:310-20.

7. Burney J, Irwin HJ. Shame and guilt in women with eating-disorder symptomatology. J Clin Psychol. 2000;56:51-61.

8. Byrne S, Wade T, Hay P, Touyz S, Fairburn C, Treasure J, Schmidt U, McIntosh V, Allen K, Fursland A, Crosby R. A randomised controlled trial of three psychological treatments for anorexia nervosa. Psychol Med. 2017:1-11. https://doi.org/10.1017/S0033291717001349. [Epub ahead of print] PubMed PMID:28552083.

9. Campbell M. Drop-out from treatment for the eating disorders: a problem for clinicians and researchers. Eur Eat Disord Rev. 2009;17:239-42.

10. Carter J, Aime A, Mills J. Assessment of bulimia nervosa: a comparison of interview and self-report questionnaire methods. Int J Eat Disord. 2001;30:187-92.
11. Carter J, McIntosh V, Jordan J, Porter R, Frampton C, Joyce P. Psychotherapy for depression: a randomized clinical trial comparing schema therapy and cognitive behavior therapy. J Affect Disord. 2013;151:500-5.

12. Cooper Z, Cooper P, Fairburn C. The validity of the eating disorder examination and its subscales. Br J Psychiatry. 1989;154:807-12.

13. Craig RJ. Overview and status of the Millon clinical multiaxial inventory. J Pers Assess. 1999;72:390-406.

14. Fairburn CG, Beglin SJ. Assessment of eating disorders: interview or self-report questionnaire? Int J Eat Disord. 1994;16:363-70.

15. Fairburn CG, Cooper Z. The eating disorder examination (twelfth edition). In: Wilson CGFGT, editor. Binge eating: nature, assessment and treatment. New York: Guilford Press; 1993. p. 317-60.

16. Fairburn CG, Cooper Z, Doll HA, O'Connor ME, Bohn K, Hawker DM, Palmer RL. Transdiagnostic cognitive-behavioral therapy for patients with eating disorders: a two-site trial with 60-week follow-up. Am J Psychiatr. 2009;166: 311-9.

17. Fairburn CG, Harrison PJ. Eating disorders. Lancet. 2003:36:407-16. https://doi.org/10.1016/S0140-6736(03)12378-1.

18. Fairburn CG, Norman PA, Welch SL, O'Connor ME, Doll HA, Peveler RC. A prospective study of outcome in bulimia nervosa and the long-term effects of three psychological treatments. Arch Gen Psychiatry. 1995;52:304-12.

19. Farrell JM, Shaw IA, Webber MA. Schema-focused approach to group psychotherapy for outpatients with borderline personality disorder: a randomized controlled trial. J Behav Ther Exp Psychiatry. 2009;40:317-28.

20. Fassino S, Pierò A, Tomba E, Abbate-Daga G. Factors associated with dropout from treatment for eating disorders: a comprehensive literature review. BMC Psychiatry. 2009;9:67-75. https://doi.org/10.1186/1471-244X-9-67.

21. Giesen-Bloo J, van Dyck R, Spinhoven P, van Tilburg W, Dirksen C, van Asselt T, Arntz A. Outpatient psychotherapy for borderline personality disorder. Randomized trial of schema-focused therapy versus transference-focused psychotherapy. Arch Gen Psychiatry. 2006;63:649-658.

22. Giles TR, Young RR, Young DE. Case studies and clinical replication series: behavioral treatment of severe bulimia. Behavioral Therapy. 1985;16:393-405.

23. Hay P. A systematic review of evidence for psychological treatments in eating disorders: 2005-2012. Int J Eat Disord. 2013;46(5):462-9.

24. Heinberg L, Thompson J, Stormer S. Development and validation of the sociocultural attitudes towards appearance questionnaire. Int J Eat Disord. 1995:17:81-9.

25. Hoffart A, Sexton H, Hedley LM, Wang CE, Holthe H, Haugum JA, Holte A. The structure of maladaptive schemas: a confirmatory factor analysis and a psychometric evaluation of factor-derived scales. Cogn Ther Res. 2005;29: 627-44.

26. Keel P, Mitchell J. Outcome in bulimia nervosa. Am J Psychiatr. 1997;154 313-21.

27. Kristeller J, Baer R, Quillian-Wolever R. Mindfulness based approaches to eating disorders, in Mindfulness-Based Treatment Approaches, ed Baer R. A. editor. San Diego: Elsevier; 2006;75-91.

28. Lawson R, Waller G, Lockwood R. Cognitive content and process in eatingdisordered patients with obsessive-compulsive features. Eat Behav. 2007;8: 305-10.

29. Lenzenweger MF. Stability and change in personality disorder features: the longitudinal study of personality disorders. Arch Gen Psychiatry. 1999;56: 1009-15.

30. Leung N, Waller $\mathrm{G}$, Thomas $\mathrm{G}$. Outcome of group cognitive-behaviour therapy for bulimia nervosa: the role of core beliefs. Behavior Research and Therapy. 2000;38:145-56

31. Lobbestael J, Van Vreeswijk M, Spinhoven P, Schouten E, Arntz A. Reliability and validity of the short schema mode inventory (SMI). Behaviour Cognitive Psychotherapy. 2010;38:437-58.

32. Luce $\mathrm{KH}$, Crowther JH. The reliability of the eating disorder examination-selfreport questionnaire version (EDE-Q). Int J Eat Disord. 1999;25:349-51.

33. Luck A, Waller G, Meyer C, Ussher M, Lacey $\mathrm{H}$. The role of schema processes in the eating disorders. Cogn Ther Res. 2005;29:717-32.

34. Malogiannis I, Arntz A, Spyropoulou A, Tsartsara E, Aggeli A, Karveli S. Schema therapy for patients with chronic depression: a single case series study. J Behav Ther Exp Psychiatry. 2014;45:319-29. https://doi.org/10.1016/j.jbtep.2014.02.003.

35. Masley S, Gillanders D, Simpson S, Taylor M. A systematic review of the evidence base for schema therapy. Cogn Behav Ther. 2012;41:182-202. https://doi.org/10.1080/16506073.2011.614274.

36. Millon T. Millon clinical multiaxial inventory-III. Minneapolis, MN: National Computer Systems; 1994. 
37. Mond JM, Hay PJ, Rodgers B, Owen C. Eating disorder examination questionnaire (EDE-Q): norms for young adult women. Behavior Research and Therapy. 2006;44: 53-62.

38. Mountford V, Waller G. Using imagery in cognitive-behavioral treatment for eating disorders: tackling the restrictive mode. Interntional Journal of Eating Disorders. 2006:39:533-43.

39. Raes F, Pommier E, Neff K, Van Gucht D. Construction and factorial validation of a short form of the self-compassion scale. Clinical Psychology \& Psychotherapy. 2011;18:250-5.

40. Rafaeli E, Bernstein DP, Young JE. Schema therapy. London: Routledge; 2011.

41. Renner F, Arntz A, Leeuw I, Huibers MJH. Treatment of chronic depression using schema therapy. Clin Psychol Sci Pract. 2013;20:166-80.

42. Renner F, Arntz AR, Peeters FPML, Lobbestael J, Huibers MJH. Schema therapy for chronic depression: results of a multiple single case series. J Behav Ther Exp Psychiatry. 2016;51:66-73.

43. Shapiro JR, Berkman ND, Brownley KA, Sedway JA, Lohr KN, Bulik M. Bulimia nervosa treatment: a systematic review of randomized controlled trials. Int J Eat Disord. 2007:40:321-36.

44. Simpson S, Morrow E, van Vreeswijk M, Reid C. Group schema therapy for eating disorders: a pilot study. Front Psychol. 2010;1:1-10.

45. Smeets $\mathrm{E}, \mathrm{Neff} \mathrm{K}$, Alberts $\mathrm{H}$, Peters M. Meeting suffering with kindness: effects of a brief self-compassion intervention for female college students. J Clin Psychol. 2014;70:794-807. https:/doi.org/10.1002/jclp.22076.

46. Thompson-Brenner H, Eddy KT, Franko DL, Dorer DJ, Vashchenko M, Kass AE, Herzog DB. A personality classification system for eating disorders: a longitudital study. Compr Psychiatry. 2008:49:551-60.

47. Tylka TL, Subich M. Examining a multidimensional model of eating disorder symptomatology among college women. J Couns Psychol. 2004:51:314-28.

48. Van Vreeswijk M, Broersen J. Schema-focused therapy: working in groups (manual). Bohn Stafleu van Loghum, Houten; 2006.

49. Waller G, Kennerley H, Ohanian V. Schema-focused cognitive behaviour therapy with eating disorders. In: Riso LP, du Toit PT, Young JE, editors. Cognitive schemas and core beliefs in psychiatric disorders: a scientist practitioners' guide. New York: American Psychiatric Association; 2007. p. 139-75.

50. Welburn K, Coristine M, Dagg P, Pontefract A, Jordan S. The schemaquestionnaire short form: factor analysis and relationship between schemas and symptoms. Cogn Ther Res. 2002;26:519-30.

51. World Health Organisation: Regional Office for Europe. Well-being measures in primary health care: the DepCare project. Stockholm: Consensus meeting; 1998.

52. Young, J., \& Brown, G. (2003). Young Schema Questionnaire: Short form. http://www.schematherapy.com/id54.htm. Accessed Jan 2015.

53. Young JE. Cognitive therapy for personality disorders: a schema focused approach. 3rd ed. Sarasota, FL: Professional Resource Exchange; 1990/1999.

\section{Submit your next manuscript to BioMed Central and we will help you at every step:}

- We accept pre-submission inquiries

- Our selector tool helps you to find the most relevant journal

- We provide round the clock customer support

- Convenient online submission

- Thorough peer review

- Inclusion in PubMed and all major indexing services

- Maximum visibility for your research

Submit your manuscript at www.biomedcentral.com/submit

C) Biomed Central 